\title{
Effects from RF spoiling disequilibrium in the background offsets of phase-contrast velocity imaging
}

\author{
Peter D Gatehouse ${ }^{1 *}$, Andreas Greiser ${ }^{2}$, David N Firmin ${ }^{1}$ \\ From 15th Annual SCMR Scientific Sessions \\ Orlando, FL, USA. 2-5 February 2012
}

\section{Summary}

To investigate how disequilibrium of spoiling affects background offset errors in phase-contrast velocity images.

\section{Background}

Phase-contrast imaging normally uses gradient-echoes with RF spoiling and phase-encode rewinding with a fixed dephasing by gradient "spoiler" pulses in each TR (time between RF pulses) studied in detail (1) where effective artifact suppression in magnitude images required $>\approx 8$ PI dephasing over the slice thickness, with $>\approx 2$ PI dephasing over an FE pixel. However, the effectiveness of spoiling depends on establishing a steadystate. In phase-contrast imaging, background offsets are known to stabilise in continuous scanning such as retrospective gating, but sequence interruptions can be necessary eg in navigator-gating or slice-tracking. Unsteady background offsets are generally ascribed to disturbed equilibrium in eddy-current effects. This abstract is a first investigation of contributions from disequilibrium in spoiling.

\section{Methods}

To separate the two sources of unsteady background, the RF pulses in a non-segmented prospectively-triggered 50 -frame cine phase-contrast $1 \mathrm{~m} / \mathrm{s}$ sequence were disabled in frames 21-30 while the gradient waveforms continued for those frames. Therefore data from frames 1-20 ("pre" RF interruption) include both sources while frames 31-50 ("post") contain only spoiling disequilibrium effects. Background velocity offsets in muscle and

\footnotetext{
${ }^{1}$ Royal Brompton Hospital, London, UK

Full list of author information is available at the end of the article
}

fat were measured at $3 \mathrm{~T}$ as functions of flip angle (20 $\left.30^{\circ}\right)$, spoiler gradients $(50,100,150 \%$ of the values in ref. 1) and TR (4.2 - 13.1ms).

\section{Results and discussion}

The different muscle and fat variations (Figure 1) imply incomplete spoiling, since eddy currents would affect both equally. Furthermore, the variations also occur post RF interruption. The results in Table 1 show: stronger variations in fat than muscle ; pre variations slightly larger than post ; no impact of greater spoiler amplitude; variations decrease sharply with lower flip angle and decrease with longer TR. A similar effect was observed in cardiac imaging (Figure 1b). Averaging was not used for the results, but was used in Figure 1 to display the effect clearly. Further work is needed to determine if the transition to equilibrium for RF spoiling can be optimised for phase-contrast imaging, and also what impact this effect has on flow measurements and whether it also occurs in blood. Thorough investigation of the optimum flip angle in phase-contrast imaging is also indicated.

\section{Conclusions}

Most of the results are consistent with incomplete spoiling in the early frames of a cine as the spoiling equilibrium is established, for example the longer T2 of fat explains its larger signals in higher-order pathways, which are reduced by lower flip angle. This effect stabilizes after a longer series of continuous gradient and RF activity, and only marginally exceeds random noise in muscle at lower flip-angles. However, for some phasecontrast sequences this effect may be significant in the background offset.

(c) 2012 Gatehouse et al; licensee BioMed Central Ltd. This is an open access article distributed under the terms of the Creative 


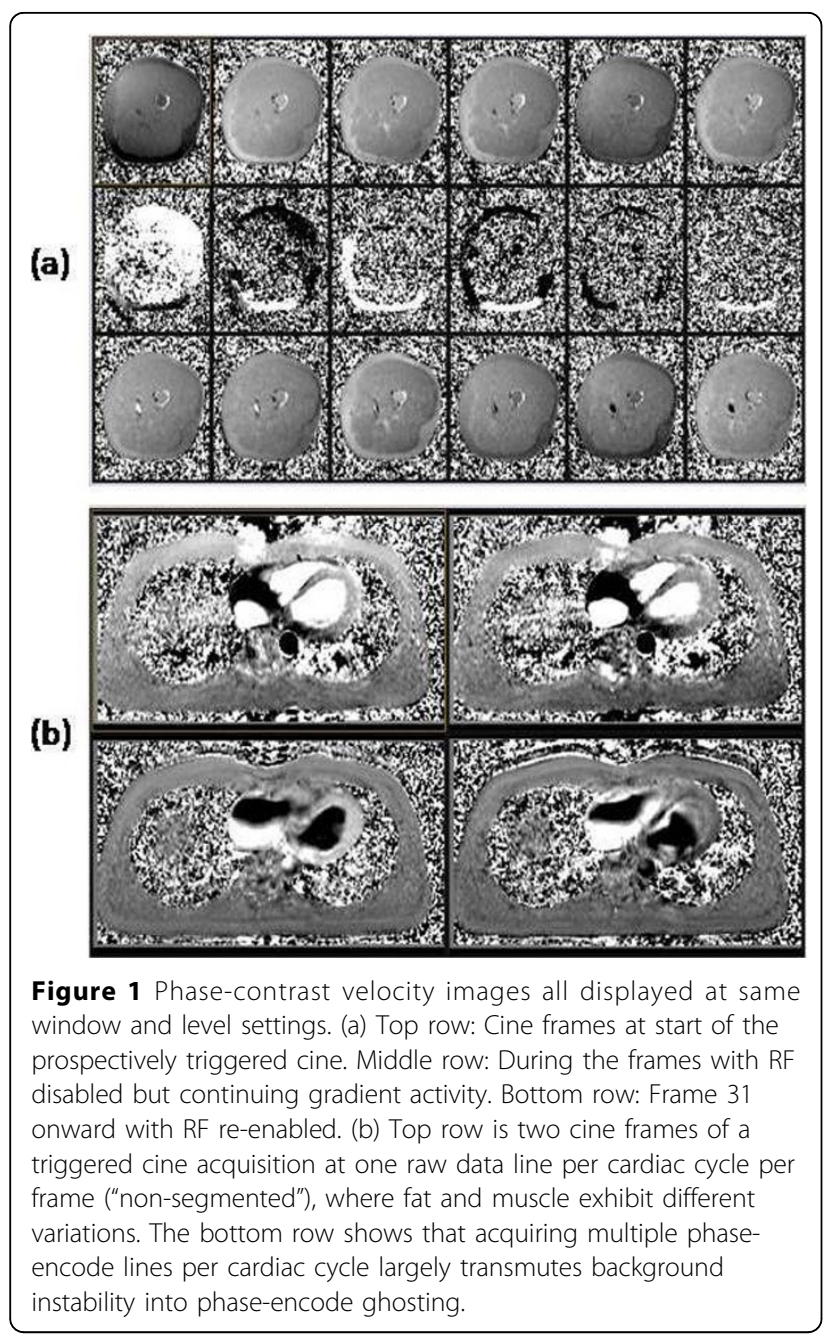

\section{Table 1 Temporal stdev $(\mathrm{cm} / \mathrm{s})$ of tissue ROls}

\begin{tabular}{ccccc}
\hline & Pre & Pre & Post & Post \\
\hline Conditions & Muscle & Fat & Muscle & Fat \\
FA30,SP150\% & 0.51 & 1.29 & 0.26 & 0.87 \\
FA30,SP100\% & 0.47 & 1.3 & 0.27 & 0.87 \\
FA30,SP50\% & 0.47 & 1.34 & 0.26 & 0.85 \\
FA20,SP150\% & 0.25 & 0.64 & 0.18 & 0.51 \\
FA20,SP100\% & 0.26 & 0.63 & 0.17 & 0.5 \\
FA20,SP50\% & 0.23 & 0.67 & 0.22 & 0.5 \\
TR4.2ms & 0.35 & 0.56 & 0.29 & 0.48 \\
TR5.6ms & 0.27 & 0.61 & 0.17 & 0.48 \\
TR8.2ms & 0.21 & 0.48 & 0.22 & 0.43 \\
TR10.7ms & 0.2 & 0.22 & 0.2 & 0.41 \\
TR13.2ms & 0.2 & 0.26 & 0.13 & 0.38 \\
\hline
\end{tabular}

Pre=Temporal stdev over Frames 1-20. Post=Temporal stdev over Frames 31 50. Frames 21-30 had no RF but continued gradients. The FlipAngle and SpoilerAmplitude tests were all at TR5.6ms. The TR tests were all at FA20 SP100\%.The temporal stdev noise level in muscle was approx $0.2 \mathrm{~cm} / \mathrm{s}$.

\section{Funding}

NIHR Cardiovascular Biomedical Research Unit funding.

\section{Author details}

'Royal Brompton Hospital, London, UK. ${ }^{2}$ Siemens Medical Systems, Erlangen, Germany.

Published: 1 February 2012

\section{Reference}

1. Leupold, Hennig, Scheffler : 2008.

\section{doi:10.1186/1532-429X-14-S1-W56}

Cite this article as: Gatehouse et al.: Effects from RF spoiling

disequilibrium in the background offsets of phase-contrast velocity imaging. Journal of Cardiovascular Magnetic Resonance 2012 14(Suppl 1): W56.

\section{Submit your next manuscript to BioMed Central} and take full advantage of:

- Convenient online submission

- Thorough peer review

- No space constraints or color figure charges

- Immediate publication on acceptance

- Inclusion in PubMed, CAS, Scopus and Google Scholar

- Research which is freely available for redistribution 